\title{
Mechanists Must be Holists Too! Perspectives from Circadian Biology
}

\author{
William Bechtel \\ Deparment of Philosophy and Center for Circadian Biology \\ University of California, San Diego
}

\begin{abstract}
The pursuit of mechanistic explanations in biology has produced a great deal of knowledge about the parts, operations, and organization of mechanisms taken to be responsible for biological phenomena. Holist critics have often raised important criticisms of proposed mechanistic explanations, but until recently holists have not had alternative research strategies through which to advance explanations. This paper argues both that the results of mechanistic strategies has forced mechanists to confront ways in which whole systems affect their components and that new representational and modeling strategies are providing tools for understanding these effects of whole systems upon components. Drawing from research on the mechanism responsible for circadian rhythms in mammals, I develop two examples in which mechanistic analysis is being integrated into a more holist perspective: research revealing intercellular integration of circadian mechanisms with those involved in cell metabolism and research revealing that stable rhythms are dependent on how individual cells in the suprachiasmatic nucleus synchronize with each other to generate regular rhythms. Tools such as network diagramming and computational modeling are providing means to integrate mechanistic models into accounts of whole systems.
\end{abstract}

Keywords: Mechanistic explanations; holism; diagrams; network diagrams; computational models; circadian rhythms; metabolism

\section{Introduction}

The strategy of explaining living phenomena by (1) linking them to specific biological systems construed as mechanisms and (2) decomposing these mechanisms into component parts and operations, and (3) offering an account of how these parts work together to produce the phenomenon has generated enormous bodies of knowledge. ${ }^{1}$ Attempts to explain physiological processes such as circulation of the blood yielded knowledge of organs like the heart where the muscles in the chambers pump the blood and valves work together to constrain its flow. Efforts to explain cellular phenomena such as fermentation resulted in knowledge of intermediary metabolites and enzymes and how operations are

${ }^{1}$ For philosophical accounts of the approach of explaining biological phenomena by characterizing mechanisms thought to be responsible for them, see Bechtel and Richardson (1993/2010); Bechtel (2006); and Machamer, Darden, and Craver (2000). 
linked to capture energy in ATP. Research directed at explaining the inheritance and expression of traits generated knowledge of genes and of molecules that play regulatory roles in gene expression. Biologists who advocated strategies of associating biological processes with biological mechanisms and then decomposing these into component parts and operations were often referred to as mechanists. Coleman's (1971) Biology in the Nineteenth Century and Allen's (1979) Life Science in the Twentieth Century document how mechanists pursued their research agenda through the $19^{\text {th }}$ and first two-third of the $20^{\text {th }}$ century. That pursuit continues today.

Mechanists, however, were not without their critics, as both Coleman and Allen make clear. Through the $18^{\text {th }}$ and $19^{\text {th }}$ century the critics of mechanistic biologists were often referred to as vitalists. Some vitalists appealed to such things as vital forces in advancing their alternative to mechanism, but many simply emphasized perceived insufficiencies of the accounts advanced by mechanists. In the $20^{\text {th }}$ century critics of the mechanists often presented themselves as holists or organicists. I shall simply group all of these critics under the label holists. A common theme among those adopting any of these labels is the view that the phenomena of life are due to the whole living organisms, not isolable mechanisms. They frequently noted that the parts of an organism function differently when in the organism than when transferred to an experimental preparation outside the organism. Often the holists were right that mechanistic accounts were inadequate to account for important aspects of biological phenomena. While holists were often adept at discovering and characterizing phenomena that the mechanists failed to account for, until recently (e.g., with the development of computational and dynamical modeling techniques) they lacked a research program to develop alternative explanations. Appealing to the need to consider the whole organism, not separable components, as holists often did, did not suffice to generate a competing explanation.

Drawing on theoretical ideas and mathematical tools that began to be developed earlier in the $20^{\text {th }}$ century by cyberneticists and general systems theorists (Green \& Wolkenhauer, 2013), towards the end of the century and on into the $21^{\text {st }}$ century biologists who often identify themselves as systems biologists have begun to develop accounts of how large, integrated systems are involved in the production of various biological phenomena. The drive to develop these new techniques is not generally motivated by an ideological commitment, as it may have been for some holists, but by the growing recognition of many mechanistic researchers of the limitations of mechanistic strategies on their own. ${ }^{2}$ Much mechanistic research sought to identify relatively simple mechanisms of few components whose operations could be characterized in linear mathematics and were organized "from start or set-up to finish or termination conditions." This description forms a central part of Machamer, Darden, and Craver's (2000) philosophical characterization of mechanisms; they acknowledge that many biological mechanisms involve feedback loops and operate non-sequentially, but they assume that such complications can be accommodated by

${ }^{2}$ Some systems biologists, however, bring with them a strong commitment to mechanistic approaches, developing models that include all the known parts and operations (see, for example, Westerhoff, Verma, Bruggeman, Kolodkin, Swat, Hayes, Nardelli, Bakker, \& Snoep, 2011). 
relatively straightforward extensions of traditional mechanistic accounts. However, the complications to what I have elsewhere characterized as basic mechanistic explanations (Bechtel, 2011) have proven far more challenging to accommodate and have led mechanists themselves to seek alternative explanatory frameworks. These alternatives enable researchers to represent and reason about the effects of components as they are integrated into whole complex systems.

I will illustrate this development within recent biology by focusing on circadian rhythm research. Circadian rhythms are rhythms endogenously generated in many organisms that have a temperature-compensated period of approximately 24-hours that can be entrained to the light-dark cycle of the local environment. Reports of 24-hour oscillations in the folding of plant leaves stem from antiquity, but demonstrating that these rhythms are endogenous and not a response to the light-dark cycle in the environment required sequestering plants from any environment clues. De Mairan (1729) did this by putting them in a dark cupboard and observing that their leaves still opened and closed on a regular cycle. In the $20^{\text {th }}$ century researchers began to study various animals, including fruit flies, mice, and humans, in constant environmental conditions and demonstrated rhythms using various behavioral and physiological measures. These studies showed rhythms with periods that were approximately, but not exactly 24 hours (circa + dies). Had the rhythm been driven by light-dark cues from the environment, they should have been exactly 24 hours; the fact that the period varied slightly from 24-hours made it clear that these oscillations were not responses to the environmental light-dark cycle.

A graph format known as an actogram provides a powerful way to visualize circadian phenomena. Figure 1 represents the activity of a mouse that was initially maintained on a regime of 12 hours light and 12 hours dark (LD), indicated by the white and black bars at the top of the actogram. Each line represents two days, with the second day repeated as the first day on the next line. Hash marks indicate periods of activity. This actogram shows that when the mouse was maintained on a LD regime, it regularly started its activity at the beginning of the dark phase, with activity diminishing towards the end of the dark phase. After seven days, mouse was shifted to continuous darkness (DD). Now each day the it began activity somewhat earlier. This is the crucial evidence that the rhythms were endogenously generated. 


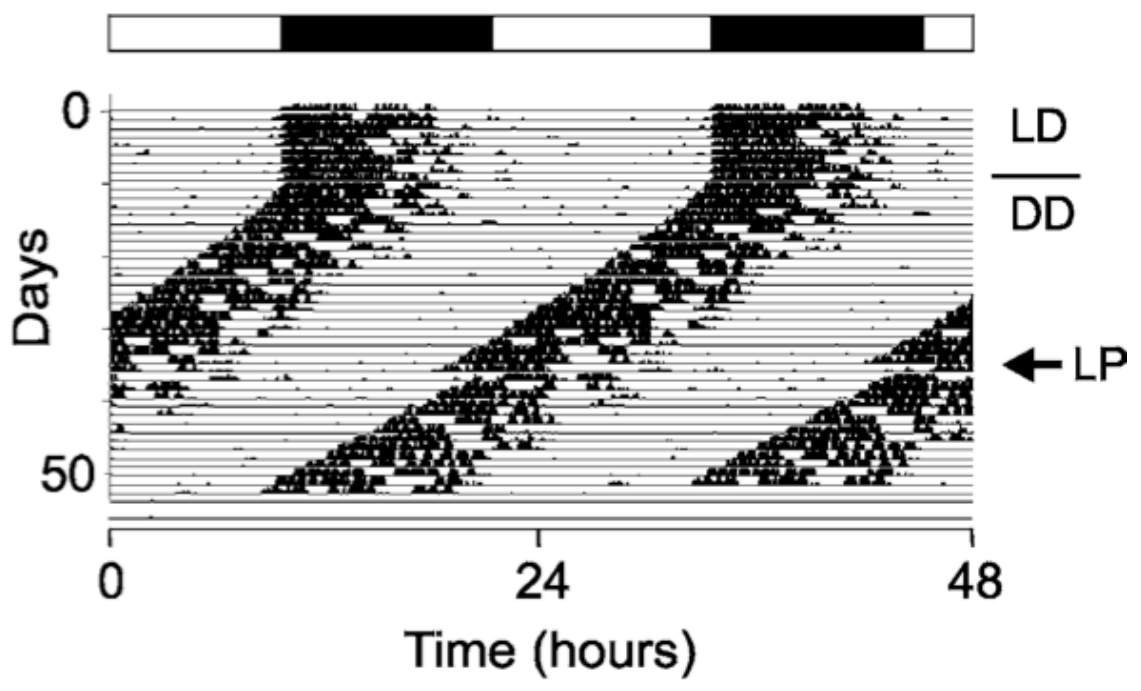

Figure 1. Actogram from Lowrey and Takahashi (2004). See text for details.

By the time of the first international conference devoted to circadian rhythms, the Symposium on Biological Clocks held at Cold Spring Harbor in 1960, the question of whether daily oscillations in behavior were generated endogenously was regarded as settled. Many of the investigations of circadian rhythms in the immediately following years focused on how these oscillations could be entrained by signals such as light. In Figure 1 the label LP identifies a day on which a four-hour light pulse was administered. The onset of activity is delayed several hours, and that delay is maintained over subsequent days, reflecting entrainment to the light pulse. However, as the title of the symposium suggests, circadian researchers were not just interested in characterizing circadian phenomena, including entrainment, but in understanding the mechanisms responsible for circadian rhythms. The term clock referred to the putative mechanism.

\section{Decomposing and Recomposing an Intracellular Circadian Mechanism}

Since mechanistic accounts try to explain biological phenomena in terms of the parts and operations of a mechanism, mechanistic research emphasizes decomposing the mechanism. The first candidate part for the circadian mechanism was a gene identified by Konopka and Benzer's (1971) through generating mutations in fruit flies that resulted in altered circadian rhythms. In this process they discovered a gene they named period (per) that, depending on how it was altered, resulted in flies that exhibited rhythms with shorter or longer than normal periods or became arrhythmic. Fruit flies typically eclode just around dawn, and when kept in total darkness, continue to eclode at a time that corresponds approximately to when dawn would have occurred. Figure 2A shows the number of eclosions per hour in a population of normal (wide type) flies and in different mutant populations. Despite being in total darkness, the normal population exhibits a large increase in eclosions at the beginning of each day. The population marked arrhythmic exhibits no daily pattern, while the flies in the short-period population eclode earlier each day and those in the long-period population eclode later each day. Until the introduction of cloning in the 1980s, however, researchers could offer no account of how per contributed to circadian rhythms. Using cloning and quantitative measures for concentrations of per 
mRNA, Hardin, Hall, and Rosbash (1990) established that in both wild type and short period flies, per mRNA also oscillates with the same period as eclosion in the fly, with the mRNA reaching its peak several hours before the protein (Figure 2B). The fact that the mRNA oscillates in a circadian fashion suggested that the gene expression process might itself be involved in generating the behavioral rhythms.

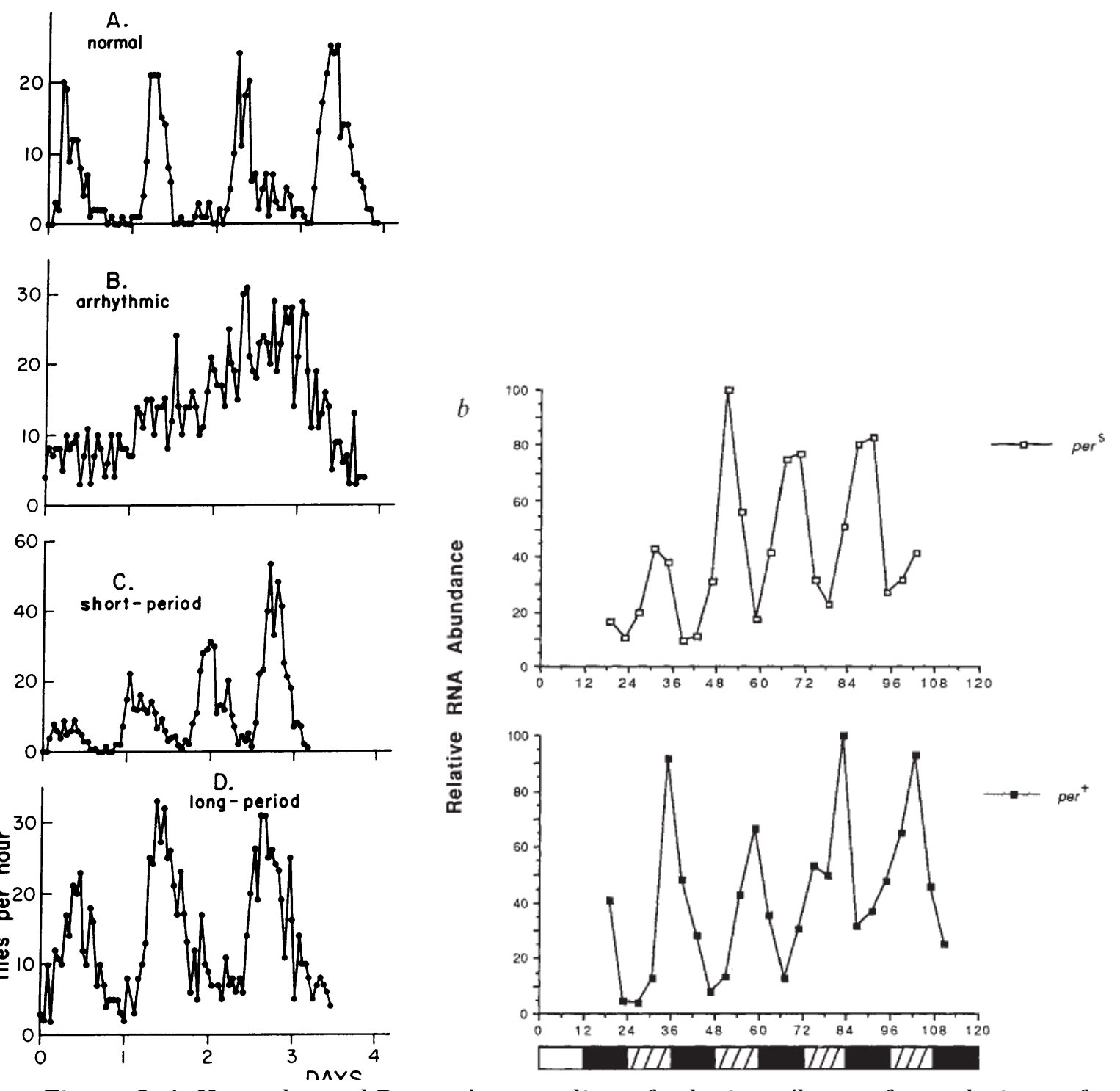

Figure 2. A. Konopka and Benzer's recording of eclosions/hour of populations of normal flies and those with three different mutations in the per gene. The second panel shows mutant flies that are arrhythmic, whereas the bottom two panels show mutant flies with short-period and long-period rhythms. B. Hardin, Hall, and Rosbash's graph of RNA abundance of per mRNA (measured by sacrificing mice and taking Western Blots) at successive time points. The mice were maintained in DD conditions, with the times indicated by hash marks indicating what would have been the light period. The top graph shows short-period mutants while the bottom graph shows wild-type flies. 
Identifying a part (per) and an operation (transcription into mRNA), however, does not provide a mechanistic account. Since the behavior of the mechanism of interest is the generation of oscillations, researchers needed to provide an account of how the transcription of per might fit into the organization of a mechanism that could oscillate. Aware that negative feedback is a mode of organization that can result in oscillations, ${ }^{3}$ Hardin et al. proposed that per figured in a negative feedback loop in which per is transcribed into mRNA, translated into a protein, with the protein feeding back in some way to inhibit its own transcription or translation (this design became known as a transcription-translation feedback loop, or TTFL). The question marks in their diagram of the proposed mechanism, (Figure 3A), reflect the fact that their proposal was only a sketch ${ }^{4}$ of a mechanism. Hardin et el. left it uncertain whether it was the protein itself, a product generated from the protein, or an a activity of the organism (perhaps waking versus sleeping), that constitutes the signal that is fed back and whether this feedback inhibits transcription or only translation. One part of filling in this sketch was to identify other components of the mechanism. For example, from the amino acid sequence of the PER protein, researchers determined that it could not directly inhibit its own transcription since it lacked a DNA binding region. This led researchers to search for other genes that, when mutated, altered circadian rhythms. One of those, discovered first in mice and named Clock $^{5}$ (Circadian Locomotor Output Cycles Kaput) by Vitaterna, King, Chang, Kornhauser, Lowrey, McDonald, Dove, Pinto, Turek, and Takahashi (1994), could bind to DNA, and PER was then inferred to function by impairing the binding of CLOCK to the promoter on the per gene. Per was soon found to have three homologues in mammals while Clock has a homologue in fruit flies, supporting the idea that PER and CLOCK performed their operations in a common mechanism. Many other genes were soon identified, some of which had homologous genes in flies and mammals, while others were unique to one or the other. Building upon this empirical research identifying component genes and evidence about how their products regulated the expression of other genes, by approximately 2005 researchers on the mammalian clock had advanced an account of a mechanism involving at least three feedback loops, as shown in Figure 3B. The figure does not show the various kinases thought to be involved in phosphorylating several of the proteins, thereby targeting them for degradation or for transport back into the nucleus where they can affect transcription of genes.

${ }^{3}$ Negative feedback is one of the first examples of what are now frequently referred to as design principles in systems biology-organizing designs whose effects can be analyzed in general and then invoked in particular instances (Green, Levy, \& Bechtel, 2014). 4 See Machamer, Darden, and Craver (2000); (Green, Levy, \& Bechtel, 2014) for the distinction between sketches and schemas.

5 The conventions for writing the names of genes differ in different research communities: fruit fly researchers usually write gene names in lower-case italics while mammalian researchers usually maintain the italics but capitalize the first letter. I follow that custom here. Protein names are typically written in capitalized Roman letters. 


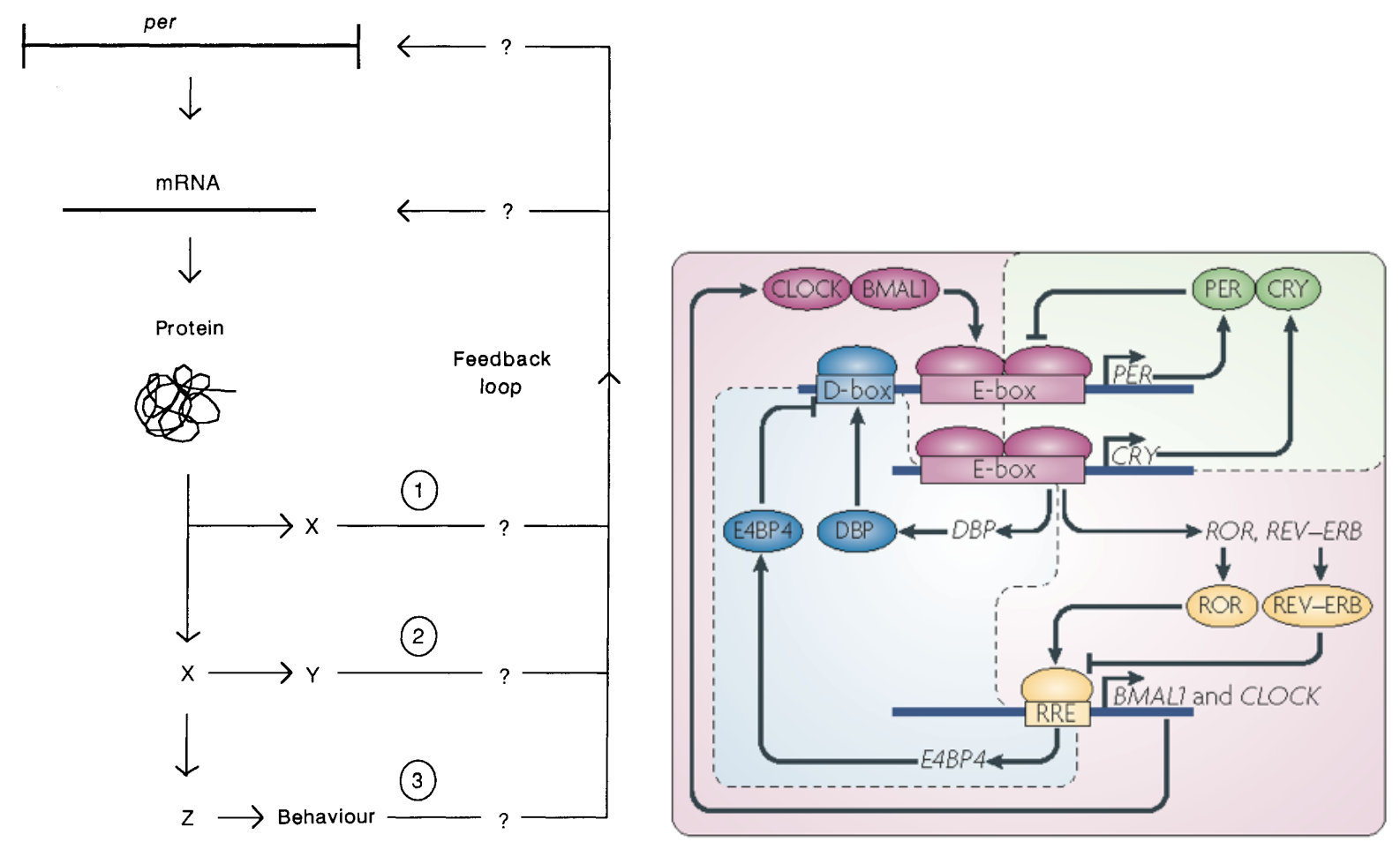

Figure 3. (A) The sketch of the transcription-translation feedback loop hypothesis advanced by Hardin et al. in 1990. (B) A much more filled in account of the clock mechanism schema presented by Zhang and Kay (2010) to reflect the conception of the clock mechanism that had been advanced by 2005 . The originally identified feedback loop involving PER is shown in the upper right, with the addition that PER forms a dimer with CRY, generated through a similar feedback loop. The PER and CRY loops are integrated with other feedback loops, most notably a positive feedback loop, shown around the bottom and left sides of the figure, in which ROR activates the expression of BMAL1, which then activates the transcription of PER and CRY. The background coloring is used to highlight three separate feedback loops.

Network diagrams such as those in Figure 3 represent attempts to recompose a mechanism from its parts by showing how different components act upon each other. An interesting feature of these diagrams is that while arrows characterize how the operations of individual components affect other components, which requires time, time is not explicitly represented. And yet the phenomenon the mechanism is supposed to explain is a sustained 24-hour oscillation in the concentrations of the various proteins from which a signal can be generated that can regulate other physiological and behavioral activities. Although negative feedback is a design principle that can yield oscillation, what is crucial to circadian rhythms is that these oscillations be sustained. With many feedback systems, oscillations will dampen over time. To establish that a TTFL as proposed by Hardin et al. or as supplemented by subsequent researchers could generate sustained oscillations, researchers turned to computational modeling. One approach to such modeling, relying on a Hill coefficient $n$ in the first equation below to represent the negative feedback, was developed by Goodwin (1965) well before the clock mechanism was proposed. In his 
schema (Figure 4A), which was inspired by Jacob and Monod's discovery of the lac-operon, a gene generated an mRNA $X$, which then was expressed in a protein $Y$, which then catalyzed a reaction producing the repressor $Z$, which then inhibited expression of the gene. For the equations shown in Figure $4 \mathrm{~B}$ to produce sustained oscillations in computational simulations, $n$ had to be unrealistically large (require too many molecules to be involved in the inhibitory process), but other modelers showed that by replacing the decay terms for each component (e.g., - $\mathrm{k}_{4} \mathrm{X}$ ) with a non-linear decay term based on Michelian-Menton kinetics $\left(k_{4} \frac{X}{K+X}\right)$, much lower and more biologically plausible Hill coefficients sufficed.

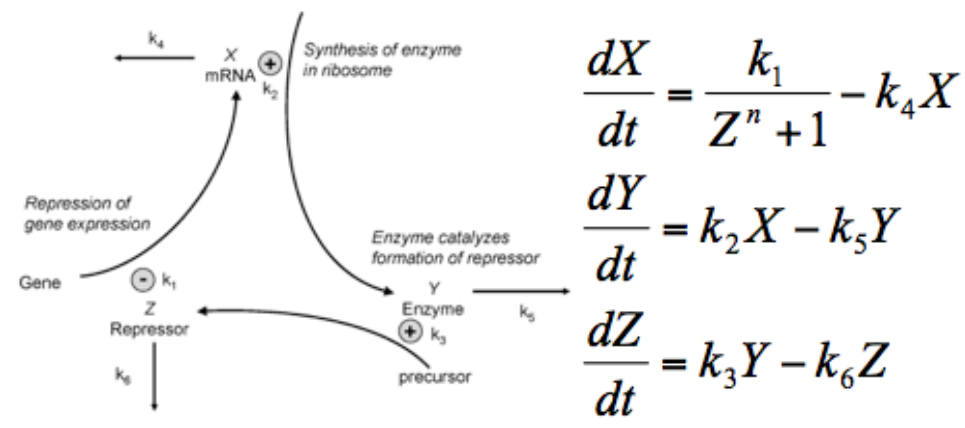

Figure 4. A. Design of a mechanism for the regulation of gene expression that provided the basis for Goodwin's computational model, and the equations of his model.

Goodwin's model was only loosely grounded in any actual mechanism - the operon was not itself advanced as a mechanism to generate oscillation, and could be viewed as at best a possible mechanism for generating oscillations. However, once Harden et al. had advanced the TTFL model in Figure 3A, Goldbeter (1995) developed a variant on these equations to show in simulation that Hardin et al.'s mechanism could generate sustained circadian rhythms. When such how-possibly models are built upon the established empirical results about both components (per and its mRNA), they serve to show that the empirically identified mechanism is capable of generating the phenomenon. The behavior of computational models depends heavily on the parameters used (the $k$ 's shown in the equations of Figure 4). At the point when Goldbeter developed his model it was not possible to use measured values for the parameters, but he makes a point of commenting that the parameters he employed fell within the range regarded as biologically plausible. The model was not just a speculative model, as was Goodwin's, but drew upon the empirical knowledge available. It also went beyond that knowledge in the attempt to establish that the proposed mechanism could produce the phenomenon those proposing it sought to explain. The sensitivity of Goldbeter and his colleagues to grounding models on what is known empirically is exemplified by that fact that as additional components and feedback loops were identified in the subsequent decade, he added equations to represent the different components that had been added. He conducted simulations with them to show that the mechanisms the experimental researchers were proposing could generate 
circadian rhythms and capture other circadian phenomena such as entrainment and, when altered, circadian pathologies (Leloup \& Goldbeter, 2008). ${ }^{6}$

Starting from Konopka's identification of the per gene, the project of decomposing the circadian clock mechanism has resulted in the characterization of many clock components and accounts of the operations they performed in the circadian clock. Since the goal was to explain circadian timekeeping, it was necessary to recompose these into an account of the whole mechanism and to demonstrate that the characterized mechanism could generate circadian phenomena. Mechanism diagrams such as those exhibited in Figure 3 played a central role in the first task while computational models played a crucial role in showing that the proposed mechanism could generate circadian rhythms. The very success of mechanistic research in identifying a multitude of parts motivated these attempts to diagrammatically and computationally recompose a much larger-scale mechanism than that with which the result had begun. The resulting explanatory schemes, advanced in network diagrams and computational models, while going beyond the experimental data on which they were based, are grounded in this evidence. The combination of empirical evidence, diagramming connections, and simulation offers stronger support for counting these proposals as mechanistic explanations than the underlying empirical evidence alone.

\section{Integrating the Intracellular Circadian Mechanism with other Intracellular Mechanisms}

When one thinks of a biological clock as a mechanism, one tends to think of it as bounded in the same manner as a mechanical clock. As a result, it is taken to be largely unaffected by activities going on outside it. Although the cell itself and its organelles are systems bounded by membranes that regulate access to the inside, cells and organelles don't correspond to individual mechanisms. Multiple mechanisms can reside in the same structure, and some mechanisms extend across these structures. The circadian clock provides an example. While bounded within individual cells, the TTFL involves transcription, a nuclear process, and translation, a cytoplasmic process, and operations that transport molecules between the nucleus and cytoplasm. (Below we will see that clocks are not restricted to single cells.) The choice of Per, Clock, and their respective genes, mRNAs, and proteins as parts of the clock mechanism, mRNAs, and proteins is partly dependent on scientists' decisions as to what they take to be central to the mechanism. Such a decision is heuristic in nature, and if it is productive, may result in an account of mechanism that can account for the phenomenon to a first approximation. But inevitably it detaches the mechanism from other biological entities with which the parts of the mechanism interact; ultimately if a more adequate account is desired, these need to be fit into it (Bechtel, 2015). In this section I will briefly describe how research on circadian rhythms has rapidly expanded to link parts of the circadian mechanism to other cellular mechanisms.

${ }^{6}$ Craver distinguished how possibly from how actually accounts of mechanisms and tends to be dismissive of the value of how possible accounts. Yet they have a critical role to play even when researchers think they have identified the actual mechanism since the identification of the mechanism does not show that the mechanism could generate the phenomenon. 
Research on circadian rhythms was motivated by the fact that circadian oscillations were found to regulate a host of behavioral and physiological processes. Initially, output signals, referred to as the hands of the clock, were thought to regulate these activities. As discussed in the next section, early research viewed only cells in one region of the mammalian brain, the suprachiasmatic nucleus (SCN) as maintaining circadian rhythms. After clock genes were discovered, though, they were found in a very wide range of tissues (Zylka, Shearman, Weaver, \& Reppert, 1998). When viewed on a populational level there was no detectable circadian rhythm in these cells when they were not connected to the SCN. However, it was eventually recognized that concentrations of clock genes do oscillate in individual cells but, without the SCN, fail to synchronize with each other (Welsh, Yoo, Liu, Takahashi, \& Kay, 2004). ${ }^{7}$ Since they possessed the machinery of the clock, cells in many tissues are now recognized as playing a role in maintaining circadian rhythms.

Within cells in different tissues, Hogenesch, Panda, Kay, and Takahashi (2004) found that a substantial number of other genes (approximately $10 \%$ of the genes, but different ones in genes in different tissues) are expressed in a circadian fashion. These genes are involved in a host of other cell activities, including metabolism and there is now a rich body of data showing various ways in which cellular metabolism is regulated by circadian rhythms (Jordan \& Lamia, 2013). What is of special interest here, though, is the fact that the metabolic system also alters the functioning of the circadian clock in peripheral tissues (and perhaps the SCN) and that there are several points of immediate connection between metabolic mechanisms and the circadian clock. I will describe two of them. Both are shown in Figure 5. In the upper left the core clock mechanism is shown around a box marked $E$ box. Two ovals representing the proteins CLOCK and BMAL1 are shown binding to the Ebox, which regulates a number of genes, indicated by the box marked gene that is connected to the E-box by a line. Curved lines extend from the gene box link to ovals for protein products, which are then shown as feeding back positively or negatively on BMAL1. The rest of the figure shows various metabolic processes regulated by the clock mechanism and pathways through which products of metabolism have effects on components of the clock itself.

${ }^{7}$ Other research has now shown that clocks in other tissues can be synchronized without the SCN by a variety of Zeitgebers such as temperature or feeding schedules (reviewed in Venkataraman, Ballance, \& Hogenesch, 2013). 


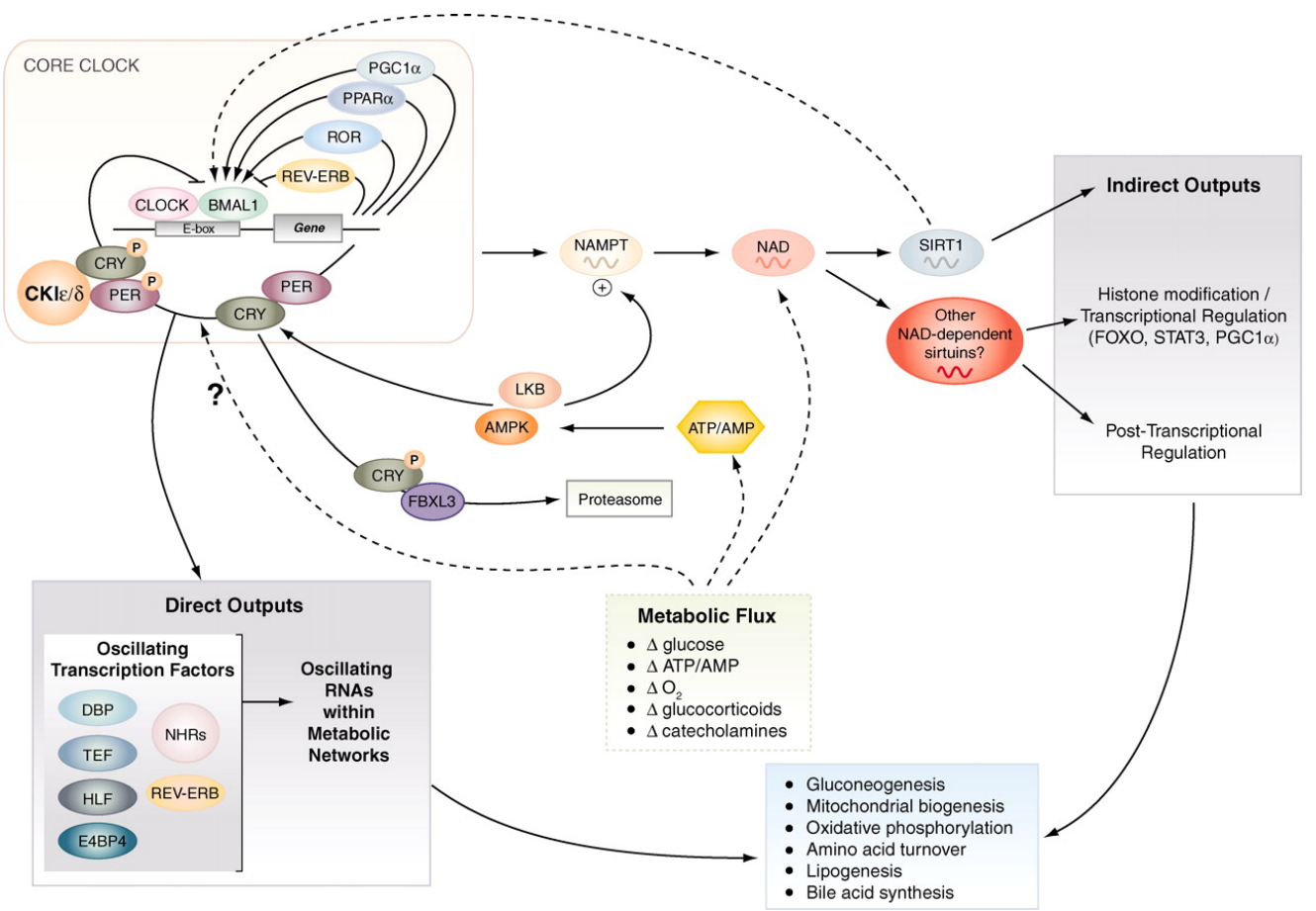

Figure 5. Network of interactions between the core clock mechanism represented in the upper left and metabolic processes that are regulated by the clock and themselves modulate clock behavior. From Bass and Takahashi, 2010

One connection through which metabolism regulates the clock mechanism involves AMPactivated protein kinase (AMPK), shown near the center for Figure 5. AMPK becomes more active, and so registers energy stress, as the concentration of ATP in the cell diminishes. AMPK regulates numerous metabolic operations by enhancing them when ATP levels are low. In particular, Lamia, Sachdeva, DiTacchio, Williams, Alvarez, Egan, Vasquez, Juguilon, Panda, Shaw, Thompson, and Evans (2009) demonstrated that AMPK phosphorylates CRY1 and CRY2. In the normally functioning core clock mechanism CRY1 and CRY2 form dimers with PER1 and PER2 respectively. These dimers then figure in the crucial feedback loop that ultimately suppresses PER and CRY synthesis. By phosphorylating CRY1 and CRY2, AMPK thus contributes to degrading CRY1 and CRY2 and thereby reduces their inhibitory effects. Jordan and Lamia (2013) suggest a second pathway by which AMPK may affect the clock mechanism: it affects the activity of the members of the casein kinase 1 class, which function in the core clock mechanism to phosphorylate PER. Although admitting that the evidence is not conclusive, Jordan and Lamia view these two roles of AMPK as evidence that it plays a central role in advancing or retarding circadian clocks in peripheral tissues.

A second path through which metabolism regulates the clock involves silent mating type information regulation 2 homolog 1 (SIRT1), shown near the right in Figure 5. SIRT1 functions as a histone deacetylase, which also deacetylates transcription factors and coregulators. Its deacetylase activity depends on the coenzyme $\mathrm{NAD}^{+}$and since the $\mathrm{NAD}^{+} / \mathrm{NADH}$ ratio reflects the cell's redox state, SIRT1's activity itself reflects the cell's energy metabolism. Nakahata, Grimaldi, Sahar, Hirayama, and Sassone-Corsi (2007) established that the protein CLOCK is a histone acetyltransferase, and that SIRT1 counters 
its activity. The acetylation and deacetylation of CLOCK affects its ability to bind to the Ebox on various circadian clock genes including Per, and hence to promote their transcription. Thus, via SIRT1, the redox state of a cell alters circadian rhythms.

In addition to these separate effects on the clock mechanism, AMPK and SIRT1 themselves interact: AMPK enhances NAD+ levels which regulates SIRT1's acetylation activity while SIRT1 can phosphorylate AMPK (Ruderman, Julia Xu, Nelson, Cacicedo, Saha, Lan, \& Ido, 2010). Thus, individually and together AMPK and SIRT1 provide linkages between what have been traditionally viewed as distinct cellular mechanisms. This is only one of many examples of linkages that have recently been established whereby other cellular processes, which are themselves regulated by the clock, also modulate the clock (Venkataraman, Ballance, \& Hogenesch, 2013). Using small interfering RNAs to screen the whole genome, Zhang, Liu, Hirota, Miraglia, Welch, Pongsawakul, Liu, Atwood, Huss, Janes, Su, Hogenesch, and Kay (2009) identified more than 200 genes in addition to the core clock genes that are both expressed in a circadian manner and that affect the amplitude or period of circadian rhythms. The differentiation of mechanisms, which has been guiding assumption of much mechanistic research, is giving way to a holist view in which multiple mechanisms in the cell each modulate others. In this case, the move to embracing the integration of mechanisms in the whole cell stems largely from strategies that were designed to identify parts of separate mechanism. The success in applying these strategies has had the side effect of revealing that the parts of different mechanisms are far more integrated than anticipated.

\section{Integrating Intracellular Operations into an Intercellular Network}

The research discussed so far has all focused on molecular processes within individual cells and addressed the need and challenge to understand an integrated set of molecular operations within cells responsible for circadian rhythms that includes operations of molecules usually assigned to other mechanisms. As research was proceeding on discovering the parts and operations responsible for the generation of circadian oscillations within cells, other research was pointing to the effects of the network in which an individual cell is contained in modulating activity within that cell. In a study to be discussed below, Welsh, Logothetis, Meister, and Reppert (1995) showed that individual SCN neurons generate circadian rhythms, but that maintaining a precise rhythm such as is found in the behavior of organisms requires the interaction of multiple cells. More recently it has become clear that circadian rhythms are affected by interactions between organs in the body and by interactions between organisms and their environments. Rather than take up these more extended networks in which circadian rhythms are situated, I will focus on research on cell-cell interactions as this suffices to illustrate the need for a holist perspective that integrates intracellular processes into a larger network.

In the same period when Konopka, working on fruit flies, discovered the first clock gene, per, Moore, working with mice, identified the SCN, a pair of nuclei in the hypothalamus (each comprising about 10,000 neurons in mice), as the locus of the clock mechanism. The SCN is located above the optic chiasm and receives direct projections from the retina, providing a pathway by which the clock could be entrained by light in the organism's 
environment (Moore, 1973). Moore and Eichler (1972) also demonstrated that when the SCN is lesioned, mice become arrhythmic. At the time there was uncertainty as to whether circadian rhythms in mammals resulted from molecular processes within individual cells, such as discussed in the previous section, or from an interaction of multiple cells organized together to generate oscillations. Welsh et al. (1995) established that circadian rhythms are a cell autonomous activity by dispersing SCN neurons onto a microelectrode array and showing continued oscillation. But they also demonstrated that the periods of the individual oscillators vary dramatically (ranging from 21.25 to 26.25 hours with a SD of 1.2 hours). As a result of the variability in period, individual neurons are soon out of phase with each other so that, as at the population level there is no detectable rhythm.

Although in Welsh et al.'s preparation a substantial number of neural connections remained, in a tissue explant from the SCN even more are retained. Herzog, Aton, Numano, Sakaki, and Tei (2004) showed that over a seven-day interval the SD of Per1 expression in explants was much less than the SD for the firing rate of dispersed neurons and closely approximated that of the running wheel behavior in the intact mouse. As shown in panels A and B of Figure 6, using a luciferase knock-in under the Per2 gene in SCN slices dispersed SCN neurons, Liu, Welsh, Ko, Tran, Zhang, Priest, Buhr, Singer, Meeker, Verma, Doyle, Takahashi, and Kay (2007) provided a compelling demonstration of the contrast in synchronization obtained between a SCN slice and dispersed neurons. These images show that in slices luciferase activity is synchronized but in dispersed preparations it is not. Panels C and D are raster plots in which each line represents a single neuron and red indicates activity above and green activity below mean activity level. Panel C shows that the activity of the individual neurons in a slice is synchronized whereas D shows neurons still oscillating (shifting from red to green) but out of phase with each other. Panels $\mathrm{E}$ and F are Raleigh plots in which blue triangles indicate the phase of individual neurons. In $\mathrm{E}$ the triangles are closely grouped whereas in F they are distributed around the whole range of phases. The arrow in Panel E indicates the average phase and the arc of the 95\% confidence interval for mean phase. Together, these graphics make clear that the SCN as a whole behaves differently than do its individual cells when they are not incorporated in the SCN. This requires that the individual cells change their behavior when they are part of the whole. How can biologists understand such behavior? 

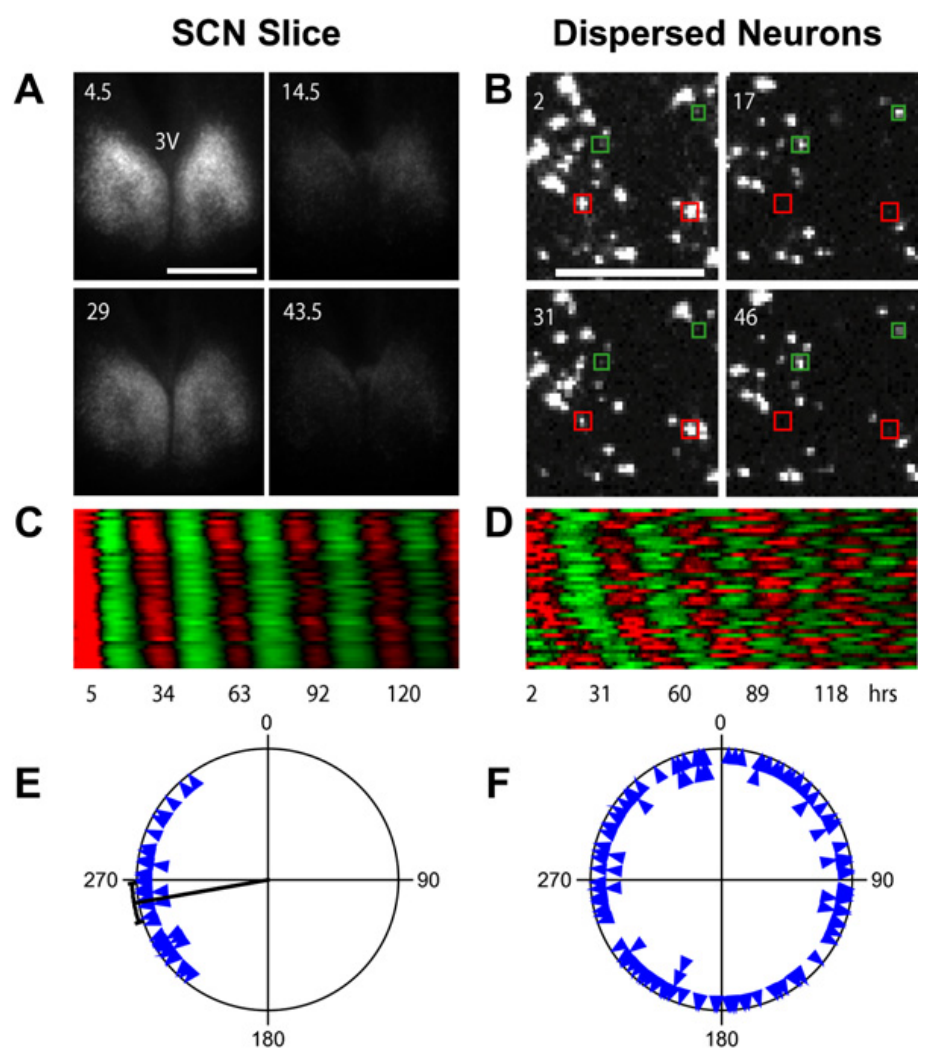

Figure 6. Three ways of contrasting the behavior of SCN neurons in slice and when dispersed. See text for details. From Liu et al. (2007).

Since the SCN simply is a nucleus of neurons, the ability of neurons to behave differently in the intact SCN than when dispersed must be due to operations through which individual neurons are affected by operations within other neurons. ${ }^{8}$ A combination of empirical research and computational modeling is beginning to provide an understanding of how this happens. In the years after Moore located circadian rhythms in the SCN, anatomical research pointed to two distinguishable subregions, a dorsomedial (shell) and a ventrolateral (core) region (van den Pol, 1980). Subsequently the expression of different hormones was associated with each area: vasoactive intestinal polypeptide (VIP) with the core and arginine vasopressin (AVP) with the shell. The phase of AVP releasing neurons in the shell was determined to be advanced over that of VIP releasing neurons in the core (van der Horst, Muijtjens, Kobayashi, Takano, Kanno, Takao, Wit, Verkerk, Eker, Leenen, Buijs, Bootsma, Hoeijmakers, \& Yasui, 1999). On the other hand, only neurons in the core received inputs from the retina so as to be able to be entrained by light. More recent studies by Welsh, Takahashi, and Kay (2010) used a luciferase knock-in attached to the Per2 gene to visualize gene expression activity in a whole coronal slice of a mouse SCN over

8 Before the TTFL mechanism had been advanced, Enright (1980) proposed the idea that the circadian system might achieve its high precision (varying only 3 to 5 minutes per day) through synchronization of multiple less precise "sloppy" oscillators. He arrived at this conclusion by comparing the precision of the circadian system with that of other biological oscillators that show much greater variability in their period. 
seven days and found a complex dynamic wave pattern in which Per2 expression begins in the shell and progresses to the core.

At present it is not possible to establish empirically the pattern of connectivity within the SCN. To evaluate various possibilities, investigators have adopted the strategy of modeling different patterns of connectivity and evaluating the plausibility of each model by how well it accounts for empirical data about how neurons synchronize in SCN slices. I briefly describe a sequence of models that have progressively provided better accounts of the empirical data and thus support more plausible hypotheses about how activity in the whole SCN is coordinated. As in many attempts to model neural connectivity, the first of these models proposed that the signal from a neuron is communicated to all other SCN neurons. Gonze, Bernard, Waltermann, Kramer, and Herzel (2005) started with the set of equations for the Goodwin model of intracellular oscillation, incorporating non-linear decay functions (using different letters used for parameters and constants) as shown in Figure 4 and in the red box in Figure 7. By using the Goodwin model rather than, for example, one of Goldbeter's models, Gonze et al. abstracted from the detail of the mechanism. However, since the critical point of their investigation is not to trace effects within individual oscillators but to show the effects of different types of coupling on synchronizing oscillators, this abstraction does not compromise their goals. They added terms to the first equation to represent the coupling between neurons (third term in the first equation) and the effect of light in entraining oscillators (final term in the first equation). A new fourth equation describes the synthesis of a neurotransmitter $V$ such as VIP from neuron $X$, which is then degenerated by the same type of Michaelian kinetics as other clock components. Assuming that the neurotransmitter is distributed to all neurons, the fifth equation calculates a mean field value $(F)$ for it. The effect of $F$ on other neurons is then represented in the third term of the first equations. Multiple simulations were performed with varying values of $K$ to represent different levels of transmitter generation. The goal was to determine how much transmitter is required to obtain synchronization. When set to 0 , representing no transmitter release, periods were normally distributed with a SD of 1.17 hours. When $K=0.5$, all cells became synchronized.

$$
\begin{aligned}
& \frac{d X_{i}}{d t}=v_{1} \frac{k_{1}^{n}}{Z_{i}^{n}+k_{1}^{n}}-v_{4} \frac{X_{i}}{k_{4}+X_{i}}+v_{c} \frac{K F}{K_{c}+K F}+L \\
& \frac{d Y_{i}}{d t}=k_{2} X_{i}-v_{5} \frac{Y_{i}}{k_{5}+Y_{i}} \\
& \frac{d Z_{i}}{d t}=k_{3} Y_{i}-v_{6} \frac{Z_{i}}{k_{6}+Z_{i}} \\
& \frac{d V_{i}}{d t}=k_{7} X_{i}-v_{8} \frac{V_{i}}{k_{8}+V_{i}} \\
& F=\frac{1}{N} \sum_{i=1}^{N} V_{i}
\end{aligned}
$$


Figure 7. Equations used in Gonze et al.'s computational model of SCN synchronization. The portion in the red box describes the activity of an isolated SCN neuron and the remainder characterizes its integration into a larger SCN network.

The Gonze et al. model showed that synchronization could be achieved by the release of VIP. These researchers did not try, however, to represent any of the known structural features of the SCN, even those these might be expected to affect how synchronization is actually achieved. In a first attempt to explore how SCN structure might affect SCN behavior, To, Henson, Herzog, and Doyle (2007) developed a more realistic model in which they employed random variations so that only about $40 \%$ of SCN neurons oscillate (corresponding to data about actual SCN neurons). They also reduced the neurotransmitter received by any cell according to the distance it is from the neuron synthesizing it, corresponding to the fact that VIP concentrations will be greatest nearest the site of release. This somewhat more realistic model still showed rapid synchronization with the introduction of the neurotransmitter. These models show that the empirically discovered peptides (VIP and AVP) could explain the capacity of SCN neurons to synchronize with each other.

The characterization of networks in these early models reflects the strategies for thinking about networks-as randomly connected or else connected into a lattice structure-that were common in the $20^{\text {th }}$ century. At the end of the century Watts and Strogratz (1998) identified an intermediate mode of network organization they referred to as small world organization. This involves most connections occurring between neighboring units and a small number of long-distance connections. This form of organization, which is increasingly recognized as ubiquitous in real-world networks, allows local clusters to be highly connected but also to be linked through only a few connections to the rest of the network. Vasalou, Herzog, and Henson (2009) revised the model of To et al. to incorporate a smallworld design and found that networks with a small percentage of long-distance connections synchronized as well as those with total connectivity. They also found that the small-world organization could account for other circadian phenomena, rendering it a plausible candidate mode of organization.

None of the models discussed so far tried to incorporate the anatomical and functional distinctions between the core and shell of the SCN that had been empirically established. In a follow-up to the Gonze et al. model, Bernard, Gonze, Čajavec, Herzel, and Kramer (2007) distinguished between core and shell regions. In particular, they supplied no direct connections between core neurons, nearest neighbor connections within the shell, and numerous projections from core neurons to ones in the shell. With this pattern of connectivity they were able to replicate the phase advance of the shell found in empirical studies. They were also able to capture effects of recovery from jet lag-with a 12-hour phase advance, the core was restored to normal activity in two days, whereas the shell required more than 10 days. In a subsequent study, Hafner, Koeppl, and Gonze (2012) explored separately the connectivity of the core and shell to see whether different connectivity patterns might generate the behavior associated with each region. They separately modeled networks with nearest neighbor, random, and scale free small-world organization. In scale-free networks, the number of connections from a node is distributed 
not in a Gaussian fashion but according to a power law so that most nodes have few connections but a few have a very large number (Barabási \& Bonabeau, 2003). Within each organizational scheme they varied whether a select group of cells or a random group received inputs from light. They then considered strategies for putting together an overall network architecture for the core, the shell, and the connections between them. They found that experimental data was best reproduced in simulations when the model used a scalefree organization with the nodes with the highest degree receiving light input in the core and a nearest neighbor organization in the shell.

The most important point, for our purposes, is that when incorporated into the SCN, individual cells behave differently. In the models this is accomplished by adding terms to the equations in models for each intracellular oscillator that capture the affects of other oscillators on a given oscillator. This approach was already illustrated in the Gonze et al. model described above; the Bernard et al. and Hafner et al. models incorporated greater detail about the model of coupling. They adopted a more detailed (but still relatively abstract) model for the intracellular oscillator that incorporated both the Per/Cry and Bmal1 feedback loops. This involved seven differential equations. They added additional equations to characterize the operation in which a neurotransmitter was synthesized from extracellular Per/Cry and the operations in which a receptor elicited a signaling cascade involving PKA and CREB. (The PKA-CREB pathway had previously been shown to activate Per and Cry transcription.) These connections are shown in Figure 8A while 8B presents the three equations added to capture the signaling process

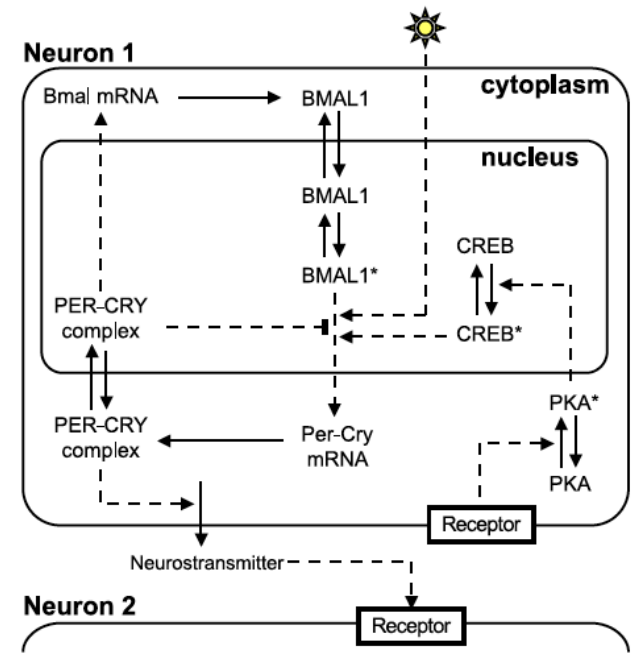

$$
\begin{aligned}
& \mathbf{u} \iota \\
& \frac{\mathrm{d}[V]}{\mathrm{d} t}=\gamma \cdot k_{21}[P E R]-k_{22}[V] \\
& \frac{\mathrm{d}\left[P K A^{*}\right]}{\mathrm{d} t}=Q(t) \cdot k_{24}\left([P K A]_{T o t}-\left[P K A^{*}\right]\right)-k_{25}\left[P K A^{*}\right] \\
& \frac{\mathrm{d}\left[C R E B^{*}\right]}{\mathrm{d} t}=k_{27}\left[P K A^{*}\right]\left([C R E B]_{T o t}-\left[C R E B^{*}\right]\right)-k_{28}\left[C R E B^{*}\right]
\end{aligned}
$$

Figure 8. (A) Hafner et al.'s diagram that supplements the core mechanism with a process for generating a neurotransmitter $(V)$, which when bound to the receptor, initiates a signaling process involving PKA and CREB.

In this section I have focused on how circadian researchers have attempted to understand the ability of individual neurons, when situated in the SCN, to synchronize their behavior with each other in the manner characterized in Figure 6. This problem has been approached by computational modeling efforts in which a model for the intracellular oscillator is supplemented by terms and equations capturing a hypothesized pattern of interaction of neurons. There is an intuitive sense in which interactions between neurons is 
at a higher level of organization than operations within individual neurons, so that if the interaction is modulating the activity of individual neurons, one is dealing with top-down effects of a higher-level system on its components. But interestingly a differentiation of levels did not figure in these accounts. Rather, the modelers simply extended the scale of their model by adding equations to capture effects on a neuron's behavior from operations outside it.

\section{On Being a Mechanist and a Holist Too}

As a result of its emphasis on decomposing systems into their parts and operations, mechanistic research is often characterized as reductionistic. Pursuit of research strategies for characterizing mechanisms and decomposing them into components has certainly been enormously productive. Until recently holist opponents of mechanistic approaches have not had equally productive ways of advancing knowledge. But the very success of mechanistic research has vindicated the need to address a central tenet of the holists' critique of mechanistic accounts - that mechanisms depends on the whole system in which they are embedded. I first illustrated this at the level of the intracellular circadian clock. The initial proposal for a mechanism required integrating per, per mRNA, and PER into a negative feedback loop. As more genes that affected circadian rhythms were identified, they needed to be incorporated into the account. And then as researchers identified links to metabolism and other cellular processes, an even more holistic account was required. Likewise, once it was shown that although maintaining circadian rhythms is a cellautonomous process, a regular and reliable circadian signal depended on synchronizing individual neurons, researchers expanded their focus beyond the cell to the whole SCN.

Two factors played a central role in facilitating the development of more holistic accounts of circadian rhythms. The first was the development of network diagrams that allowed researchers to represent the multiple interacting components. Figure 3B exemplifies a network diagram of a mechanism that tries to integrate the multiple feedback loops that are conceived of as working together in the generation of circadian rhythms within individual cells while Figure 5 exemplifies a mechanism diagram relating that core clock mechanism with other components of metabolic mechanisms. Finally, Figure 8 presents the way Hafner et al. envisaged the connections between intercellular oscillators, the generation from them of intercellular signals, and the operations through which individual cells respond to these signals. In each case, the set of operations are more complicated that can easily be represented linguistically and diagrams provide a way of representing the multiplicity of connections and enable researchers to reason about the effects of these connections.

Diagrams, however, are static structures and the mechanisms they represent are dynamical systems generating oscillations and synchronizing them. With very simple mechanisms, scientists can mentally rehearse the operations of the mechanism, but with non-linear interactions between components organized in a non-sequential manner, humans typically fail to accurately represent how the mechanism will behave. This brings us to the second factor that has made investigating whole systems possible-computational modeling. In the case of intercellular circadian rhythms, these models enable researchers to integrate 
the operations of multiple different known components and simulate the functioning of the whole mechanism. In the case of intercellular interactions, researchers modeled multiple neurons and added into the equations characterizing the known operations whereby each neuron is modulated by the activity of others.

Tools such as diagrammatic representations and computational modeling are enabling biologists to characterize and analyze the behavior of whole systems. Before modeling tools, including the computers on which to run them, were developed, there weren't tools for understanding whole integrated systems. But with these tools, biologists are able to address the phenomena emphasized by holists. With these tools for understanding mechanisms as situated in whole systems, mechanists can be holists too. Moreover, they must be if they are to make sense of the results their own research has generated.

\section{References}

Allen, G. E. (1979). Life science in the twentieth century. London ; New York: Cambridge University Press.

Barabási, A.-L., \& Bonabeau, E. (2003). Scale-free networks. Scientific American, 50-59.

Bechtel, W. (2006). Discovering cell mechanisms: The creation of modern cell biology. Cambridge: Cambridge University Press.

Bechtel, W. (2011). Mechanism and biological explanation. Philosophy of Science, 78, 533557.

Bechtel, W. (2015). Can mechanistic explanation be reconciled with scale-free constitution and dynamics? Studies in History and Philosophy of Science Part C: Studies in History and Philosophy of Biological and Biomedical Sciences.

Bechtel, W., \& Richardson, R. C. (1993/2010). Discovering complexity: Decomposition and localization as strategies in scientific research. Cambridge, MA: MIT Press. 1993 edition published by Princeton University Press.

Bernard, S., Gonze, D., Čajavec, B., Herzel, H., \& Kramer, A. (2007). Synchronization-induced rhythmicity of circadian oscillators in the suprachiasmatic nucleus. PLoS Computational Biology, 3, e68.

Coleman, W. (1971). Biology in the nineteenth century : problems of form, function, and transformation. New York: Wiley.

De Mairan, J.-J. d. O. (1729). Observation Botanique. Histoire de l'Academie Royale Sciences, 35.

Enright, J. T. (1980). Temporal precision in circadian systems: a reliable neuronal clock from unreliable components? Science, 209, 1542-1545.

Goldbeter, A. (1995). A model for circadian oscillations in the Drosophila Period protein (PER). Proceedings of the Royal Society of London. B: Biological Sciences, 261, 319324.

Gonze, D., Bernard, S., Waltermann, C., Kramer, A., \& Herzel, H. (2005). Spontaneous synchronization of coupled circadian oscillators. Biophysical Journal, 89, 120-129.

Goodwin, B. C. (1965). Oscillatory behavior in enzymatic control processes. Adv Enzyme Regul, 3, 425-438.

Green, S., Levy, A., \& Bechtel, W. (2014). Design sans adaptation. European Journal for Philosophy of Science, 5, 15-29. 
Green, S., \& Wolkenhauer, O. (2013). Tracing organizing principles: learning from the history of systems biology. History and Philososophy of the Life Sciences, 35, 553-576.

Hafner, M., Koeppl, H., \& Gonze, D. (2012). Effect of network architecture on synchronization and entrainment properties of the circadian oscillations in the suprachiasmatic nucleus. Plos Computational Biology, 8, e1002419.

Hardin, P. E., Hall, J. C., \& Rosbash, M. (1990). Feedback of the Drosophila period gene product on circadian cycling of its messenger RNA levels. Nature, 343, 536-540.

Herzog, E. D., Aton, S. J., Numano, R., Sakaki, Y., \& Tei, H. (2004). Temporal precision in the mammalian circadian system: A reliable clock from less reliable neurons. Journal of Biological Rhythms, 19, 35-46.

Hogenesch, J. B., Panda, S., Kay, S. A., \& Takahashi, J. S. (2004). Circadian transcriptional output in the SCN and liver of the mouse. In J. A. G. Derek J. Chadwick (Ed.), Molecular Clocks and Light Signalling (pp. 171-183).

Jordan, S. D., \& Lamia, K. A. (2013). AMPK at the crossroads of circadian clocks and metabolism. Molecular and Cellular Endocrinology, 366, 163-169.

Konopka, R. J., \& Benzer, S. (1971). Clock mutants of Drosophila melanogaster. Proceedings of the National Academy of Sciences (USA), 89, 2112-2116.

Lamia, K. A., Sachdeva, U. M., DiTacchio, L., Williams, E. C., Alvarez, J. G., Egan, D. F., Vasquez, D. S., Juguilon, H., Panda, S., Shaw, R. J., Thompson, C. B., \& Evans, R. M. (2009). AMPK regulates the circadian clock by cryptochrome phosphorylation and degradation. Science, 326, 437-440.

Leloup, J.-C., \& Goldbeter, A. (2008). Modeling the circadian clock: From molecular mechanism to physiological disorders. BioEssays, 30, 590-600.

Liu, A. C., Welsh, D. K., Ko, C. H., Tran, H. G., Zhang, E. E., Priest, A. A., Buhr, E. D., Singer, O., Meeker, K., Verma, I. M., Doyle, F. J., Takahashi, J. S., \& Kay, S. A. (2007). Intercellular coupling confers robustness against mutations in the SCN circadian clock network. Cell, 129, 605-616.

Lowrey, P. L., \& Takahashi, J. S. (2004). Mammalian circadian biology: Elucidating genomewide levels of temporal organization. Annual Review of Genomics and Human Genetics, 5, 407-441.

Machamer, P., Darden, L., \& Craver, C. F. (2000). Thinking about mechanisms. Philosophy of Science, 67, 1-25.

Moore, R. Y. (1973). Retinohypothalamic projection in mammals: A comparative study. Brain Research, 49, 403-409.

Moore, R. Y., \& Eichler, V. B. (1972). Loss of a circadian adrenal corticosterone rhythm following suprachiasmatic lesions in the rat. Brain Research, 42, 201-206.

Nakahata, Y., Grimaldi, B., Sahar, S., Hirayama, J., \& Sassone-Corsi, P. (2007). Signaling to the circadian clock: plasticity by chromatin remodeling. Current Opinion in Cell Biology, 19, 230-237.

Ruderman, N. B., Julia Xu, X., Nelson, L., Cacicedo, J. M., Saha, A. K., Lan, F., \& Ido, Y. (2010). AMPK and SIRT1: a long-standing partnership? American Journal of Physiology Endocrinology and Metabolism, 298, E751-E760.

To, T.-L., Henson, M. A., Herzog, E. D., \& Doyle, F. J., III. (2007). A molecular model for intercellular synchronization in the mammalian circadian clock. Biophysical Journal, 92, 3792-3803. 
van den Pol, A. N. (1980). The hypothalamic suprachiasmatic nucleus of rat: Intrinsic anatomy. The Journal of Comparative Neurology, 191, 661-702.

van der Horst, G. T. J., Muijtjens, M., Kobayashi, K., Takano, R., Kanno, S.-i., Takao, M., Wit, J. d., Verkerk, A., Eker, A. P. M., Leenen, D. v., Buijs, R., Bootsma, D., Hoeijmakers, J. H. J., \& Yasui, A. (1999). Mammalian Cry1 and Cry2 are essential for maintenance of circadian rhythms. Nature, 398, 627-630.

Vasalou, C., Herzog, E. D., \& Henson, M. A. (2009). Small-world network models of intercellular coupling predict enhanced synchronization in the suprachiasmatic nucleus. Journal of Biological Rhythms, 24, 243-254.

Venkataraman, A., Ballance, H., \& Hogenesch, J. B. (2013). The role of the circadian system in homeostasis. In A. J. M. Walhout, M. Vidal \& J. Dekker (Eds.), Handbook of Systems Biology: Concepts and insights (pp. 407-426). Amsterdam: Elsevier.

Vitaterna, M. H., King, D. P., Chang, A.-M., Kornhauser, J. M., Lowrey, P. L., McDonald, J. D., Dove, W. F., Pinto, L. H., Turek, F. W., \& Takahashi, J. S. (1994). Mutagenesis and mapping of a mouse gene, Clock, essential for circadian behavior. Science, 264, 719725.

Vogelstein, B., Papadopoulos, N., Velculescu, V. E., Zhou, S., Diaz, L. A., Jr., \& Kinzler, K. W. (2013). Cancer genome landscapes. Science, 339, 1546-1558.

Watts, D., \& Strogratz, S. (1998). Collective dynamics of small worlds. Nature, 393, 440-442.

Welsh, D. K., Logothetis, D. E., Meister, M., \& Reppert, S. M. (1995). Individual neurons dissociated from rat suprachiasmatic nucleus express independently phased circadian firing rhythms. Neuron, 14, 697-706.

Welsh, D. K., Takahashi, J. S., \& Kay, S. A. (2010). Suprachiasmatic nucleus: Cell autonomy and network properties. Annual Review of Physiology, 72.

Welsh, D. K., Yoo, S.-H., Liu, A. C., Takahashi, J. S., \& Kay, S. A. (2004). Bioluminescence imaging of individual fibroblasts reveals persistent, independently phased circadian rhythms of clock gene expression. Current Biology, 14, 2289-2295.

Westerhoff, H., Verma, M., Bruggeman, F., Kolodkin, A., Swat, M., Hayes, N., Nardelli, M., Bakker, B., \& Snoep, J. (2011). From Silicon Cellsilicon cell to Silicon Humansilicon human. In B. Booß-Bavnbek, B. Klösgen, J. Larsen, F. Pociot \& E. Renström (Eds.), BetaSys (Vol. 2, pp. 437-458): Springer New York.

Zhang, E. E., \& Kay, S. A. (2010). Clocks not winding down: unravelling circadian networks. Nature Reviews Molecular and Cell Biology, 11, 764-776.

Zhang, E. E., Liu, A. C., Hirota, T., Miraglia, L. J., Welch, G., Pongsawakul, P. Y., Liu, X., Atwood, A., Huss, J. W., Janes, J., Su, A. I., Hogenesch, J. B., \& Kay, S. A. (2009). A genome-wide RNAi screen for modifiers of the circadian clock in human cells. Cell, 139, 199-210.

Zylka, M. J., Shearman, L. P., Weaver, D. R., \& Reppert, S. M. (1998). Three period homologs in mammals: Differential light responses in the suprachiasmatic circadian clock and oscillating transcripts outside of brain. Neuron, 20, 1103-1110. 